\title{
An Investigation of Resonant Photoemission in Gd with X-Ray Linear Dichroism
}

\author{
S.R. Mishra \\ T.R. Cummins \\ G.D. Waddill \\ W.J. Gammon \\ G. van der Laan \\ K.W. Goodman \\ J.G. Tobin
}

This paper was prepared for submittal to the

American Vacuum Society Symposium

Baltimore, MD

November 2-6, 1998

July 1998

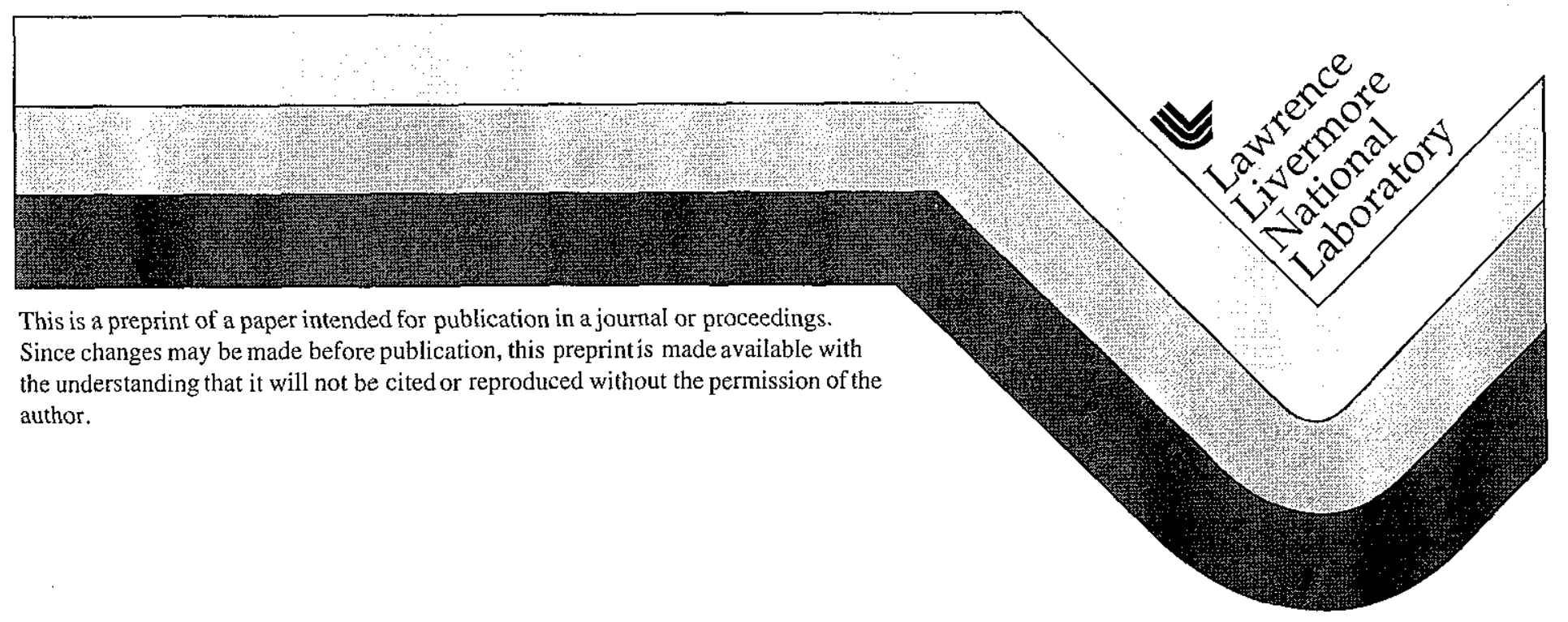




\section{DISCLAIMER}

This document was prepared as an account of work sponsored by an agency of the United States Government. Neither the United States Goverrment nor the University of California nor any of their employees, makes any warranty, express or implied, or assumes any legal liability or responsibility for the accuracy, completeness, or usefulness of any information, apparatus, product, or process disclosed, or represents that its use would not infringe privately owned rights. Reference herein to any specific commercial product, process, or service by trade name, trademark, manufacturer, or otherwise, does not necessarily constitute or imply its endorsement, recommendation, or favoring by the United States Government or the University of California. The views and opinions of authors expressed herein do not necessarily state or reflect those of the United States Government or the University of California, and shall not be used for advertising or product endorsement purposes. 
Submitted to J. Vac. Sci. Tech. in conjunction with the 1998 Symposium of the American Vacuum Society

\title{
An Investigation of Resonant Photoemission in Gd with X-Ray Linear Dichroism
}

\author{
S.R. Mishra ${ }^{1}$, T.R.Cummins ${ }^{2}$, G.D. Waddill ${ }^{2}$, W.J. Gammon ${ }^{1}$, \\ G. van der Laan ${ }^{3}$, K.W. Goodman ${ }^{4}$ and J.G. Tobin ${ }^{4}$
}

${ }^{1}$ Dept. of Physics, Virginia Commonwealth Univ., Richmond, VA, USA 23284

${ }^{2}$ Dept. of Physics, University of Missouri-Rolla, Rolla, MO, USA 65401-0249

3

Daresbury Laboratory, Warrington WA4 4AD, United Kingdom

${ }^{4}$ Lawrence Livermore National Laboratory, Livermore, CA, USA 94550

\begin{abstract}
The constructive summing of direct and indirect channels above the absorption threshold of a core level can cause a massive increase in the emission cross section, leading to a phenomenon called "resonant photoemission". Using novel magnetic linear dichroism in angular distribution photoelectron spectroscopy experiments and theoretical simulations, we have probed the nature of the resonant photoemission process in $\mathrm{Gd}$ metal. It now appears that temporal matching as well as energy matching is a requirement for true resonant photoemission.

PACS \#: $\quad 75.70 \mathrm{i} \quad$ Magnetic Films and Multilayers

79.60 -i Photoemission and Photoelectron Spectra

75.50 -y Studies of Specific Magnetic Materials
\end{abstract}

Corresponding Author: J.G. Tobin, E-mail Tobin1@LLNL.Gov 


\section{Introduction}

The "resonant photoemission" of $4 \mathrm{f}$ and $5 \mathrm{p}$ electrons from rare-earth metals and their compounds can arise when the photon has just enough energy to excite a $4 \mathrm{~d}$ electron to an unoccupied $4 \mathrm{f}$ level, leading to a strong enhancement of the intensity of the overall process. (See Fig. 1). In a generic picture, the indirect channel of the resonant photoemission is interpreted as due to a process where a $4 \mathrm{~d}$ electron in the initial state is first excited to the unoccupied $4 \mathrm{f}$ level, forming a tightly coupled, bound intermediate state, $4 \mathrm{~d}$ core hole plus $4 \mathrm{f}$ electrons. (Fig.2) Then a decay via autoionization occurs into the final state, thus producing a final state identical to that obtained by a direct photoemission process for the ejected electron. ${ }^{1}$ The transition rate is greatly enhanced if the excited state decay is by a Coster-Kronig or a super-Coster-Kronig [ (s)CK] process. 2,3 The key question is whether these processes are coherent or incoherent: Is it truly "resonant photoemission" or merely the incoherent addition of a second emission channel? Should the overall intensity be treated as a squaring of the sum of the amplitudes (coherent) or summing of the squares of the amplitudes (incoherent)? A true "resonant photoemission" process should be coherent, involving interference terms between the direct photoemission and indirect photoemission channels. Possibly, incoherence would give rise to the loss of photoemission characteristics in the process, with a domination of Augerlike properties.

The availability of synchrotron $x$-rays has added a new tool for the study of magnetism, attracting considerable interest both from experimental and theoretical point of view. The magnetic properties of surface, subsurface, and interfaces have been probed using linearly ${ }^{4-7}$ polarized light in photoemission and circularly ${ }^{8-13}$ polarized light in 
both photoemission and photoabsoption modes. The interaction of circularly or linearly polarized light with the ferromagnetic atom excite a core electron thus leaving behind a core hole. The spin-orbit coupling and the exchange interaction of the core hole with the polarized valence electrons lead to what is called the magnetic dichroism. The shape and intensity of photoemission or photoabsorption spectra excited with circularly or linearly polarized light depend on the relative orientation of photon polarization and sample magnetization. The dependence of photoemission line shapes, and thus the dichroism, on the direction of magnetization when using linearly polarized light leads to what is called the magnetic linear dichroism in the angular dependence (MLDAD). ${ }^{47,12}$ As this effect arises due to the interference between the emission channels $1+1$ and $1-1$, it specifically depends upon the emission direction and vanish under the angle integration.

MLDAD, which probes the core-levels of atom, can provide wealth of information on electronic and magnetic nature of atoms as the shape of dichroic spectra depends on the core spin-orbit interaction and the exchange interaction. MLDAD experiments have been performed on transition metal $^{4-7,14,15}$ and rare earth metals ${ }^{15}$ to understand magnetic properties of these metallic system. Herein we report the use of MLDAD effect in the $4 \mathrm{f}$ and $5 \mathrm{p}$ core-level photoemission from ferromagnetic Gd metal to investigate the nature of the "resonantly emitted" photoelectrons. The resonance effect at the $3 p$ threshold has been observed in transition metals due to $3 p-3 d$ transition. ${ }^{16}$ For the $3 \mathrm{~d}$ transition metals, the resonant enhancement is less prominent because the $3 \mathrm{~d}$ states of transition metals are less localized than the $4 \mathrm{f}$ states of rare-earths, leading to smaller matrix elements between the initial and 
the intermediate state. Thus, the attraction to study rare earth $\mathrm{Gd}$ metal comes in part from its well localized nature of the $4 \mathrm{f}$ states which allows

resonant photoemssion to be treated as an atomic process ${ }^{17}$.

To the problem of "Resonant Photoemission," we have applied the new photoelectron spectroscopy technique of magnetic linear dichroism in angular distributions (MLDAD). ${ }^{4-7,12}$ This technique is related to but distinct from the techniques of magnetic $x$ ray circular dichroism $(M X C D)$ in photoelectron spectroscopy and $x$ ray absorption. ${ }^{8-13}$ The key is that while large dichroic effects in ferromagnets can be observed with MXCD-photoemission and MXCDabsorption, the large MLDAD effects in ferromagnets is solely a photoemission, not an absorption-driven, process. This is because the chirality which gives rise to magnetic sensitivity is due to the vectorial configuration in MLDAD as opposed to the intrinsic chirality of circularly polarized x rays in the MXCD techniques. ${ }^{4-7,14}$ In absorption, where there is an essential averaging over all emission angles, the vectorial chirality is lost. Thus, MLDAD is the ideal measurement to distinguish between photoemission and absorption processes. Angle-resolved photoemission in a magnetic system should show an MLDAD effect: $x$ ray absorption and thus Auger-like emission will show no MLDAD effect. It is this test which we have applied to the "resonant photoemission" of the Gd5p and Gd4f emissions.

\section{Experimental}

The photoemission experiments were performed with linearly polarized soft $x$-ray at the undulator Beamline-7 at the Advanced Light Source Facility at Lawrence Berkeley National Laboratory. 14,18,19

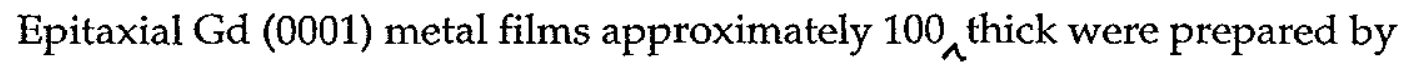
e-beam evaporation onto a $Y(0001)$ substrate at room temperature. An 
approximately 100 Angstrom film was grown because the Curie point is expected to be significantly reduced from the bulk value of $293 \mathrm{~K}$ for thin $\mathrm{Gd}$ films with thickness less than 15 monolayer (ML). ${ }^{20}$ Subsequent annealing for $45 \mathrm{sec}$ at $710 \mathrm{~K}$ resulted in well-ordered Gd films. The quality of the substrate as well as of the film was checked via $x$-ray photoelectron spectroscopy, excited using $\mathrm{Mg} \mathrm{Ka}$ and $\mathrm{Al} \mathrm{Ka}$ radiation, respectively. The crystallinity of the film was monitored via low energy electron diffraction (LEED) and $x$ ray photoelectron diffraction (XPD). ${ }^{19}$ The Gd films were magnetized remanently in plane along [11-20] by the pulses of $100 \mathrm{kOes}$ from a nearby solenoid. From previous studies of $\mathrm{Gd}(0001) / \mathrm{W}(0001)$, the magnetization of the film is expected to lie in the plane in a single domain state. ${ }^{21}$ All photoemission measurements were made in remanence at approximately $250 \mathrm{~K}$. The angle resolved photoemission spectra were collected in the chiral geometry (Fig 1(c)), using a Perkin Elmer hemispherical energy analyzer with $a+/-2$ degree acceptance angle and with the energy resolution of $250 \mathrm{meV}$ (obtained from Fermi level cut off). The photoelectron intensity is normalized to the photon flux to correct for variations in the synchrotron radiation intensity. MLDAD spectras are recorded by reversing the magnetization, by rotating the sample by 180 degrees, perpendicular to the data collection plane. In Ref 19, we have demonstrated that this is equivalent to a magnetization flip for this system.

\section{Theoretical}

Theoretical spectra were calculated in intermediate coupling using Cowan's relativistic Hartree-Fock code. ${ }^{22}$ Radiative transitions were taken into account to first order and (s)CK transitions to infinite order. ${ }^{23}$ Line broadening of the photoelectron state and experimental resolution were included by a convolution with a Lorentzian and a Gaussian, respectively. The values of the parameters can 
be found in Refs 13 and 23. For the $4 \mathrm{f}$ emission the interference effects between the different photoemission final states and between direct and resonant channel were fully included. For the $5 \mathrm{p}$ emission the interference between the direct and resonant channel was treated as scalar in order to reduce the overall size of the calculation. The interference term was excluded in the $5 \mathrm{p}$ calculation.

\section{Discussion}

Before considering the photoemission spectra, let us review the photon energy dependence in the resonance regime as evidenced in the xray absorption spectrum and non-dichroic photoemission spectra. The total electron yield spectrum from metallic Gd is displayed in Fig.1a. There is a group of weak narrow peaks near the $4 \mathrm{~d}$ absorption edge and a broad strong absorption feature at higher energy, around $150 \mathrm{eV}$, far beyond the $4 \mathrm{~d}$ absorption edge. The strong intermediate coupling resulting from the exchange and coulomb interaction between $4 \mathrm{~d}$ hole and $4 \mathrm{f}$ electrons results in multiplet splitting of the $4 \mathrm{~d}^{9} 4 \mathrm{f}^{8}$ configuration. $^{13}$ These interactions are very large due to the large radial overlap of the $4 \mathrm{~d}$ and $4 \mathrm{f}$ wave functions. Features in the $4 \mathrm{~d}-4 \mathrm{f}$ absorption curve arises from the transition from the ground state level of the $4 \mathrm{~d}^{10} 4 \mathrm{f}^{7}$ configuration to the numerous intermediate levels of $4 \mathrm{~d}^{9} 4 \mathrm{f}^{8}$ configuration. The broad maximum, or "giant resonance," arises from the rapid decay of the intermediate states from the $4 \mathrm{~d}^{9} 4 \mathrm{f}^{8}$ configuration into a continuum with an ejected electron. ${ }^{24,25}$ This type of giant resonance absorption has been observed before in partially filled $5 \mathrm{f}$, $4 \mathrm{f}$, and $3 \mathrm{~d}$ metals and their alloys and compounds. ${ }^{1}$ Figure $1(\mathrm{~b})$ shows a set of angle resolved EDC's of Gd taken in normal emission at photon energies corresponding to on and off resonance of $4 \mathrm{~d}-4 \mathrm{f}$ absorption threshold. It is evident that the strong increase in the $\mathrm{Gd} 4 \mathrm{f}$ peak photoemission intensity around $8 \mathrm{eV}$ is correlated with the onset of the Gd 4d-4f giant-resonance absorption. The REPES intensity 
increase is caused by the constructive summing of the direct photoemission channel and indirect photoemission channel. ${ }^{23}$

Now consider the $4 \mathrm{f}$ photoemission dichroism results. Figure 3 shows a set of angle resolved EDC's and difference curves. These are at photon energies corresponding to on and off resonance of the $4 \mathrm{~d}-4 \mathrm{f}$ giant absorption maximum. The resonant photoelectron spectroscopy (REPES) effects are distinguished by comparing photoemission intensity of spectra taken on $(150 \mathrm{eV})$ and off $(95 \mathrm{eV})$ resonance. Experimentally, it is evident that the fairly strong dichroism (a few $\%)$ in the Gd 4f peak photoemission intensity persists "on" and "off" resonance, despite the 5 fold increase in signal size in going from $\mathrm{hv}=95 \mathrm{eV}$ to $\mathrm{hv}=150 \mathrm{eV}$ (See the vertical scales on panels 3(a) and 3(c).) This behavior is also seen in the theoretical spectra seen in Fig. $3 \mathrm{e}$ and $3 \mathrm{~g}$. Here, a ten fold increase in intensity at resonance and a $20 \%$ dichroism is predicted. Not surprisingly, a stronger effect in resonant enhancement and dichroic behavior is found in the theoretical spectra. Experimental results are always limited by technical imperfections such as contaminants and non-ideal alignments, crystallinities, and magnetizations, as well as polarizations below $100 \%$. The REPES is caused by the constructive interference between the direct $\mathrm{PE}$ channel and the indirect photoemission channel. ${ }^{23}$ In the direct $4 \mathrm{f}$ photoemission process, an electron in the half filled $\mathrm{f}^{7}$ ground state absorbs a photon and is ejected into the continuum leaving behind an $\mathrm{f}^{6}$ ionized atom. As per conventional arguments the resonant mechanism consists of a two step process, $4 \mathrm{~d}^{10} 4 \mathrm{f}^{7}+\mathrm{hv} \rightarrow 4 \mathrm{~d}^{9} 4 \mathrm{f}^{8} \rightarrow 4 \mathrm{~d}^{10} 4 \mathrm{f}^{6}+\varepsilon \mathrm{l}$, where $\varepsilon \mathrm{l}$ represents the electron in the continuum state. (Fig. 2a). [This is related to but not the same as a typical Auger process which leaves behind a doubly ionized atom: $\left.4 \mathrm{~d}^{10} 4 \mathrm{f}^{7}+\mathrm{hv} \rightarrow 4 \mathrm{~d}^{9} 4 \mathrm{f}^{7}+\varepsilon \mathrm{l} \rightarrow 4 \mathrm{~d}^{10} 4 \mathrm{f}^{5}+\varepsilon \mathrm{l}+\varepsilon \mathrm{l}\right]$ In the first step, as the photon energy is tuned through the $4 \mathrm{~d}$ absorption edge, an electron from the $\mathrm{d}$ shell gains enough energy to be moved into an empty spin minority $4 \mathrm{f}$ state. In 
the second step, the $4 \mathrm{~d}^{9} 4 \mathrm{f}^{8}$ intermediate state decays via autoionization mechanism such that $4 \mathrm{~d}$ core-hole is refilled by an electron from the $4 \mathrm{f}$ shell and another electron is simultaneously ejected. However, the latter event taking the system to the final state cannot be treated as an individual, time-ordered energyconserving event. Quantum mechanics treats the transition between the initial and the final states as a single step process. In fact, our observation of the retention of an MLDAD effect directly confirms that this interpretation is correct. (Spectra taken over the photon energy range of 142-154 eV show similar but not identical effects. $^{17-19}$ See Fig. 4. The near identicality of the pairs of theoretical spectra ( $3 \mathrm{e}$ and $3 \mathrm{~g})$ is somewhat accidental. At other photon energies, differences arise, as can be seen in Fig. 4.) The interference between channels is necessary for the observation of photoemission dichroism in a regime where the indirect channel dominates the total cross section. So this is clearly a coherent process, as illustrated in Fig. 2C, where cross channel interference is crucial. Our observation of photoemission effects in the $4 \mathrm{f}$ emission resonance is consistent with Olson et al's report ${ }^{26}$ of valence band dispersion in the La $4 \mathrm{~d}-4 \mathrm{f}$ resonance and the observation of photoemission characteristics in Ce4f resonant emission, using angular distribution measurements. ${ }^{27}$ Also, it should be noted that the MLDAD resonant behavior is similar but not identical to the MXCDphotoemission resonance results of Starke et al (Ref. 13). Correspondingly, when we performed MXCD-photoemission at a photon energy of $95 \mathrm{eV}$, a different dichroism was observed, ${ }^{14,28}$ consistent with earlier reports. ${ }^{12,13,29}$

Next, let us consider the 5p emission shown in Fig. 5. (See Fig. $2 b$ for the channel diagram). Here, there is a large dichroism observed off resonance at $h v=137 \mathrm{eV}$, with a disappearance of any dichroism on resonance $(\mathrm{hv}=151 \mathrm{eV})$. In this case, there is a three fold increase experimentally and a ten fold increase 
theoretically in the intensity, in going from off resonance to on resonance.

Interestingly, the percentage dichroisms match very well between experiment and theory. Moreover, despite using parameters derived elsewhere, ${ }^{13,23}$ a very good match is observed between the theoretical and experimental spectra and difference curves, including all of the fine structure in the $5 p$ manifold. Over the photon energy range of $138-150 \mathrm{eV}$, other EDC pairs exhibit similar dichroic differences to that at $h v=137 \mathrm{eV}$ but with strong changes in the shapes of the "raw" EDC spectra and a decrease in the dichroism percentage (PND) as the photon energy moves toward the maximum of the giant resonance. ${ }^{30}$ The disappearance correlates with the giant resonance. Here, it is clear that the second equation in Fig. 2c applies, where the process is incoherent and emission at $h v=151 \mathrm{eV}$ is essentially auger-like, not a direct photoemission process at all.

This raises a key question: "Why is the $4 \mathrm{f}$ emission "photoemission-like" and the 5p emission "Auger-like"?" The answer may lie in the regime of time. The Coster-Kronig decay that occurs in the $5 \mathrm{p}$ emission occurs on a time scale of about $10^{-15}$ sec. $^{2}$ The super-Coster-Kronig delay of the $4 \mathrm{f}$ should be significantly faster. $^{2,3}$ This would speed up the indirect channel, bringing it nearer to the time duration of $x$ ray absorption $\left(\tau \leq 10^{-17} \mathrm{sec}\right)$ that dominates the direct photoemission channel. Thus, not only must the energies of the two channels match but also the time duration, in order to observe "true resonant photoemission". However, it is prudent at this point to offer a word of caution. While this picture seems to hold nicely for resonant emission in rare earths, or at least gadolinium, extension of this model to transition metal cases might be imprudent. Significant controversy remains concerning these other systems and it is generally accepted that there are important difference in mechanism between 
rare earth and $3 \mathrm{~d}$ transition metal resonant emission, owing to their different electron structures. $27,31-34$

Now, operating within the constraint that we are discussing only rare earth resonant emission, can we find a correlation in the parameters used to calculate the theoretical spectra with this simple temporal picture? The required parameters can be obtained directly from Cowan's calculation. However, here starts the first complication from the proposed holistic model. The Gd $4 \mathrm{~d}$ absorption spectrum consists of hundreds of different lines each having different parameters, and therefore a different coherence. Fortunately, in the case of Gd 4d edge they divide globally, and rather nicely, into two different regions (i) the preedge peaks and (ii) the giant resonance. We can deduce two things from the parameters: (a) At a given photoemission decay channel, the lifetime of the states in region (i) is about 10 to 20 times longer than in region (ii). This is due to the differences in (s)CK decay rates, as manifestly demonstrated by the strongly different line widths in the $4 \mathrm{~d}$ absorption spectrum in Fig.1. (b) For a given absorption state, the $\mathrm{SCK}$ decay to the $4 \mathrm{f}$ is about 6 times faster than the CK decay to the $5 \mathrm{p}$. Thus the $4 \mathrm{f}$ photoemission is connected to a 6 times shorter lifetime of the $4 \mathrm{~d}$ hole. If resonant photoemission dichroic interference effects occur, they will occur for the $4 \mathrm{f}$ photoemission decay, but only at the giant resonance (i.e. where the decay is fastest). Outside of the giant resonance regime, the regular photoemission dichroic effects can play a role, as seen in both the Gd $4 \mathrm{f}$ and $5 \mathrm{p}$ emissions. It is important to note the distinction between the these two regions of the $4 \mathrm{~d}$ absorption.

Conclusions 
We have investigated Gd resonant photoemission with MLDAD. This photoemission technique allows for a direct isolation of photoemission and Auger-like contributions. The $\mathrm{Gd} 4 \mathrm{f}$ resonant photoemission is confirmed to be photoemission-like. The Gd 5 p resonant emission is shown to be dominated by Auger-like contributions. Temporal channel matching is a requirement for channel interference and the persistence of photoemission effects.

\section{Acknowledgements}

The authors, particularly JGT, would like to thank David Pappas for his guidance and aid, especially in the thesis work of W.J. Gammon. This work was performed under the auspices of the U.S Department of Energy by Lawrence Livermore National Laboratory under contract no. W-7405-Eng-48. Experiments were carried out at the Spectromicroscopy Facility (Beamline 7.0) at the Advance Light Source, built and supported by the U.S. Department of Energy. We would also like to thank F.O. Schumann and R.F. Willis for aid in some of the data collection. 


\section{References}

${ }^{1}$ Extensive references by J. Allen in Synchrotron Radiation Research, ed. R.Z. Bachrach (Plenum Press, New York, 1992), Vol. 1., p. 253, Giant Resonances in Atoms, Molecules, and Solids, eds. J.P. Connerade, J.-M. Esteva, and R.C. Karnatak, NATO ASI series B (Plenum Press, New York, 1987).

${ }^{2}$ B. Feuerbacher, B. Fitton and R.F. Willis, ed., "Photoemission and the Electronic Properties of Surfaces", John Wiley \& Sons, New York, See Fig. 5.1 on Page 115.

${ }^{3}$ T.A. Carlson, "Photoemission and Auger Spectroscopy," Plenum Press, New York.

${ }^{4}$ Ch. Roth, F.U. Hillebrecht, H.B. Rose, and E. Kisker, Phys. Rev. Lett. 70, 3479 (1993); Solid State Commun. $\underline{86} 647$ (1993); F.U. Hillebrecht, Ch. Roth, H.B. Rose, W.G. Park, E. Kisker, and N.A. Cherepkov, Phys. Rev. B53, 12182 (1996); F. U. Hillebrecht, H. B. Rose, Ch. Roth, and E. Kisker, J. Magn. Magn. Mat.146, 49 (1995).

${ }^{5}$ F. Sirotti and G. Rossi, Phys. Rev. B 49, 15682 (1994); G. Rossi, F. Sirotti, N. A. Cherepkov, F. Combet Farnoux, and G. Panaccione, Solid State Commun. 90, 557 (1994).

${ }^{6}$ W. Kuch, M.T. Lin, W. Steinhogl, C.M. Schneider, D. Venus and J. Kirschner, Phys. Rev. B51, 609 (1995).

${ }^{7}$ F.O. Schumann, R.F. Willis, K.W. Goodman and J.G. Tobin, Phys. Rev. Lett. 79 $5166(1997)$.

${ }^{8}$ L. Baumgarten, C.M. Schneider, H. Petersen, F. Schafers, and J. Kirschner, Phys. Rev. Lett. 65, 492 (1990); C.M. Schneider, D. Venus, and J. Kirschner, Phys. Rev. B 45, 5041 (1992).

${ }^{9}$ J.G. Tobin, G.D. Waddill and D.P. Pappas, Phys. Rev. Lett. $\underline{68}, 3642$ (1992).

${ }^{10}$ C.M. Schneider, D. Venus, and J. Kirschner, Phys. Rev. B 45, 5041 (1992). 
B.T. Thole and G. van der Laan, Phys. Rev. B 44, 12424 (1991).

12

D. Venus, Phys. Rev. B 48, 6144 (1993); Phys. Rev. B 47, 8821 (1994).

13

K. Starke, E. Navas, E. Arenholz, Z. Hu, L. Baumgarten, G. van der Laan, C.T. Chen and G. Kaindl, Phys. Rev. B 55, 2672 (1997); K. Starke, E. Navas, L. Baumgarten, and G. Kaindl, Phys. Rev. B 48, 1329 (1993).

14

J.G. Tobin, K.W. Goodman, F.O. Schumann, R.F. Willis, J.B. Kortright, J.D.

Denlinger, E. Rotenberg, A. Warwick, and N.V. Smith, Surf. Sci. Lett. 395, 227 (1998). J.G. Tobin, K.W. Goodman, G.J. Mankey, R.F. Wills, J.D. Denlinger, E. Rotenberg, and A. Warwick, J. Appl. Phys. 79, 5626 (1996); J. Vac. Sci. Tech., B 14, 3171 (1996).

15 T. Kinoshita, H. B. Rose, C. Roth, F. U. Hillebrecht, and E. Kisker,J. Electron Spec. Related Phen. 78, 333 (1996).

16 C. Cuillot, Y. Ballu, J. Paigue, J. Ecante, K. P. Jain, P. Thiry, R. Pinchauz, Y. Petroff, and L. M. Falikov, Phys. Rev. Lett. 39, 1632 (1977), L. C. Davis and L. A. Feldkamp, Phys. Rev. A 17, 2012 (1978).

${ }^{17}$ S.-J.Oh and S. Donaich, Phy. Rev. B 26, 1859 (1982).

18

J.D. Denlinger et al. Rev. Sci. Instrum. 66, 1342 (1995).

${ }^{19}$ W.J. Gammon, et al, J.Vac. Sci. Technol. A 15, 1 (1997); S.R. Mishra, T.R. Cummins, G.D. Waddill, K.W. Goodman, J.G. Tobin, W.J. Gammon, T. Sherwood, and D.P. Pappas, J. Vac. Sci. Tech. A 16, 1348 (1998); W.J. Gammon, M.S. Thesis, Virginia Commonwealth Univ., 1996, Unpublished.

${ }^{20}$ M. Farle, K. Babershke, U. Stetter, A. Aspelmeier, and F. Gerhardter, Phys. Rev. B 47, 11571 (1993).

${ }^{21}$ A. Berger, A. W. Pang, and H. Hopster, Phys. Rev. B 52, 1078 (1995). 
22

R.D. Cowan, The Theory of Atomic Structure and Spectra, University of California Press, Berkeley (1981).

23

G. van der Laan, M. Surman, M.A. Hoyland, C.J.F. Flipse, B.T. Thole, Y. Seino, H. Ogasawara and A. Kotani, Phys. Rev. B 46, 9336 (1992); G. van der Laan, E. Arenholz, E. Navas, Z. Hu, E. Mentz, A. Bauer and G. Kaindl, Phys. Rev. B 56, 3244 (1997).

${ }^{24}$ J.L. Dehmer, A.F. Starace, U. Fano, J. Sugar, and J.W. Cooper, Phys. Rev. Lett 26, 1521 (1971); J. Sugar, Phys. Rev. B 5, 1785 (1972), A. F. Starace, Phys. Rev. B 5, 1773 (1972); L.C. Davis, and L.A. Feldkamp, Phys. Rev. A 17, 2012 (1978).

${ }^{25}$ F. Gerken, J. Barth, and C. Kunz, Phys. Rev. Lett. 47, 993 (1981).

${ }^{26}$ C.G. Olson, P.J. Benning, M. Schmidt, D.W. Lynch, P. Canfield, D. M. Wieliczka, Phys. Rev. Lett., 76, 4265 (1996)

${ }^{27}$ M.F. Lopez, A. Gutierrez, C. Laubschat, and G. Kaindl, J. Electr. Spectrosc. Relat. Phenom. 71, 73 (1995).

28 K.W. Goodman; J.G. Tobin, F.O. Schumann, R.F. Willis, J.W. Gammon, D.P. Pappas, J.B. Kortright, J.D. Denlinger, E. Rotenberg, A. Warwick, and N.V. Smith, MRS Symp. Proc. 475, 493 (1997)

${ }^{29}$ E. Arenholz, Ph.D. Thesis, "Magnetic Dichroism in Photoemission from Lanthanide Materials: Basic Concepts and Applications", Wissenschaft and Techrik Verlag, Berlin, 1996.

30

T.R. Cummins et al, under preparation.

${ }^{31}$ J.G. Tobin and G.D. Waddill, J. Appl. Phys. 75,6369 (1994). The LMM Transitions are Coster-Kronig, not Super-Coster-Kronig. 
${ }^{32}$ M.M. Traum, N.V. Smith, H.H. Farrell, D.P. Woodruff, and D. Norman, Phys. Rev. B 20, 4008 (1979)

33

M. Weinhelt, A. Nilsson, M.Magnuson, T. Wiell, N. Wassdahl, O. Karis, A. Fohlisch, N. Martensson, J. Stohr, and M. Samant, Phys. Rev. Lett. 78, 967 (1997).

${ }^{34}$ L.C. Davis, J. Appl. Phys. 59, R25 (1986).

${ }^{35}$ J.J. Yeh, and I. Lindau, At. Data and Nucl. Data Tables 32, 1 (1985). 


\section{FIGURE CAPTIONS}

Figure 1(a). An $x$-ray absorption spectrum of $G d(0001) / Y(0001)$ recorded in a total electron yield mode. The pre-peak structure occurs around photon energies of $135-143 \mathrm{eV}$ and the giant resonance is at photon energy of 148 $\mathrm{eV}$. (b) A comparison of Gd 4f photoemission spectra in non-resonant vs. $4 \mathrm{~d}-4 \mathrm{f}$ resonant photoemission. The spectrum shown with open circles was taken at off resonance with photon energy of $h v=95 \mathrm{eV}$, and the spectrum shown with filled circles was taken near the $4 \mathrm{~d}-4 \mathrm{f}$ resonant maximum at photon energy of $h v=150 \mathrm{eV}$. (c) Synchrotron radiation PES experimental geometry. The synchrotron $\mathrm{x}$-rays are incident at an angle $\mathrm{a}=30$ degrees relative to the $Y Y^{\prime}$ axis. The photoelectrons are collected along the sample normal (See Ref. 19.)

Figure 2 (a) Schematic diagram of the direct and indirect channels in Gd $4 \mathrm{f}$ Resonant Photoemission. Time estimates are based on Ref. 2 and 3. (b) Same for Gd5p emission. (c) Comparison of coherent and incoherent additions of channel contributions. $\mathrm{AD}(\mathrm{AI})$ is the direct (indirect) amplitude.

Figure 3. A series of experimental and theoretical $4 \mathrm{f}$ photoemission spectra (for the two opposite magnetization directions) and normalized difference curves. (a) $h v=150 \mathrm{eV}$, photoelectron spectra, experimental. (b) $\mathrm{hv}=150 \mathrm{eV}$, photoelectron spectra difference, experimental. (c) $h v=95 \mathrm{eV}$, photoelectron spectra, experimental. (d) $\mathrm{hv}=95 \mathrm{eV}$, photoelectron spectra difference, experimental.(e) $h v=150 \mathrm{eV}$, photoelectron spectra, theory. (f) $\mathrm{hv}=150 \mathrm{eV}$, photoelectron spectra difference, theory. (g) hv=95 eV, photoelectron spectra, theory. (h) hv=95 eV, photoelectron spectra difference, theory. EDC is energy distribution curve. The spectra in (a), (c), (e), \& (g) are EDC's, where the photon energy is held constant 
and the kinetic energy is scanned. PND stands for peak normalized difference, where the dichroism difference at each binding energy is divided by the sum of the two intensity maxima, one from each pair. (Following Refs. 4 and 19.) The photon energy of $150 \mathrm{eV}$ is "on" resonance and $95 \mathrm{eV}$ is "off" resonance. [c.f. Fig. 1.] The relative intensities of the experimental curves were determined by normalizing to the valence band intensities and then correcting for the valence band cross sections. (See Refs. 19 and 35.)

Figure 4(a). Experimental MLDAD in Gd 4d-4f REPES as the photon energy is tuned through the photoabsorption region, see figure 1. (b). Theoretical MLDAD spectra calculated in intermediate coupling scheme using Cowan's relativistic Hartee-Fock code.

Figure 5. Analogous to figure 3, with photon energies of $151 \mathrm{eV}$ and $137 \mathrm{eV}$ and looking at $5 \mathrm{p}$ emission. The photon energy of $151 \mathrm{eV}$ is "on" resonance and the $137 \mathrm{eV}$ is "off" resonance. [c.f. Fig. 1d.] 


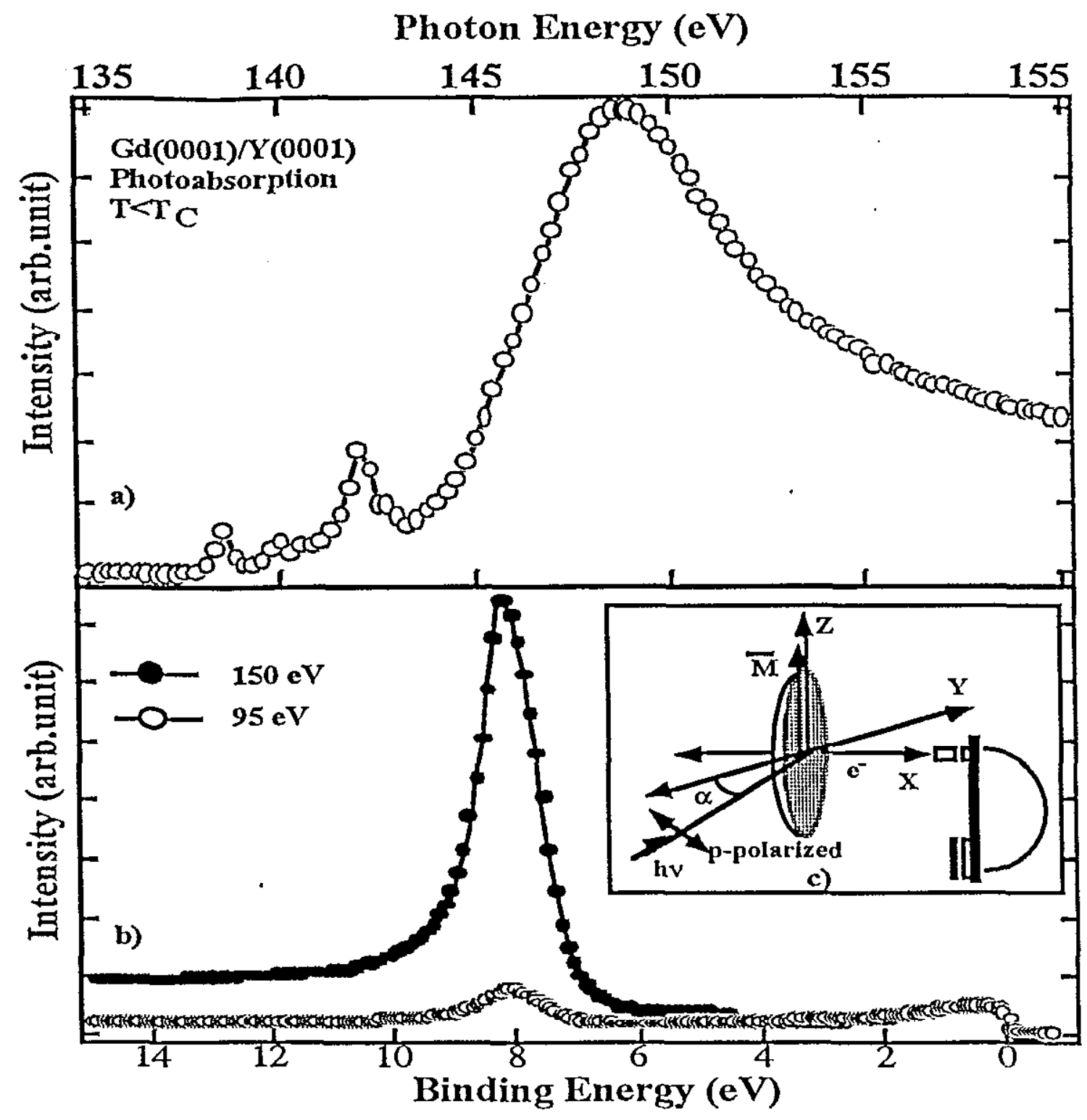

Figure 1. 


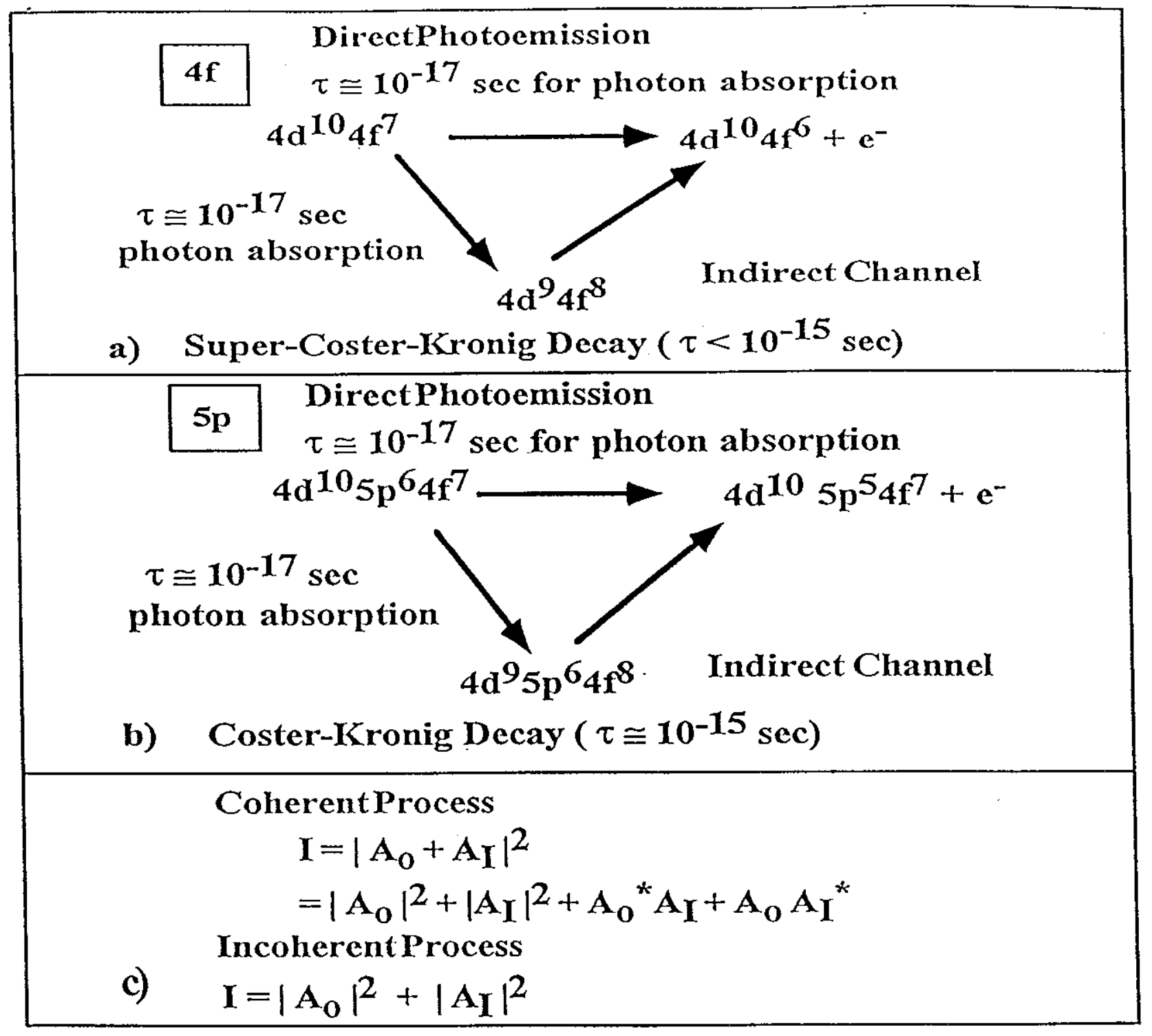




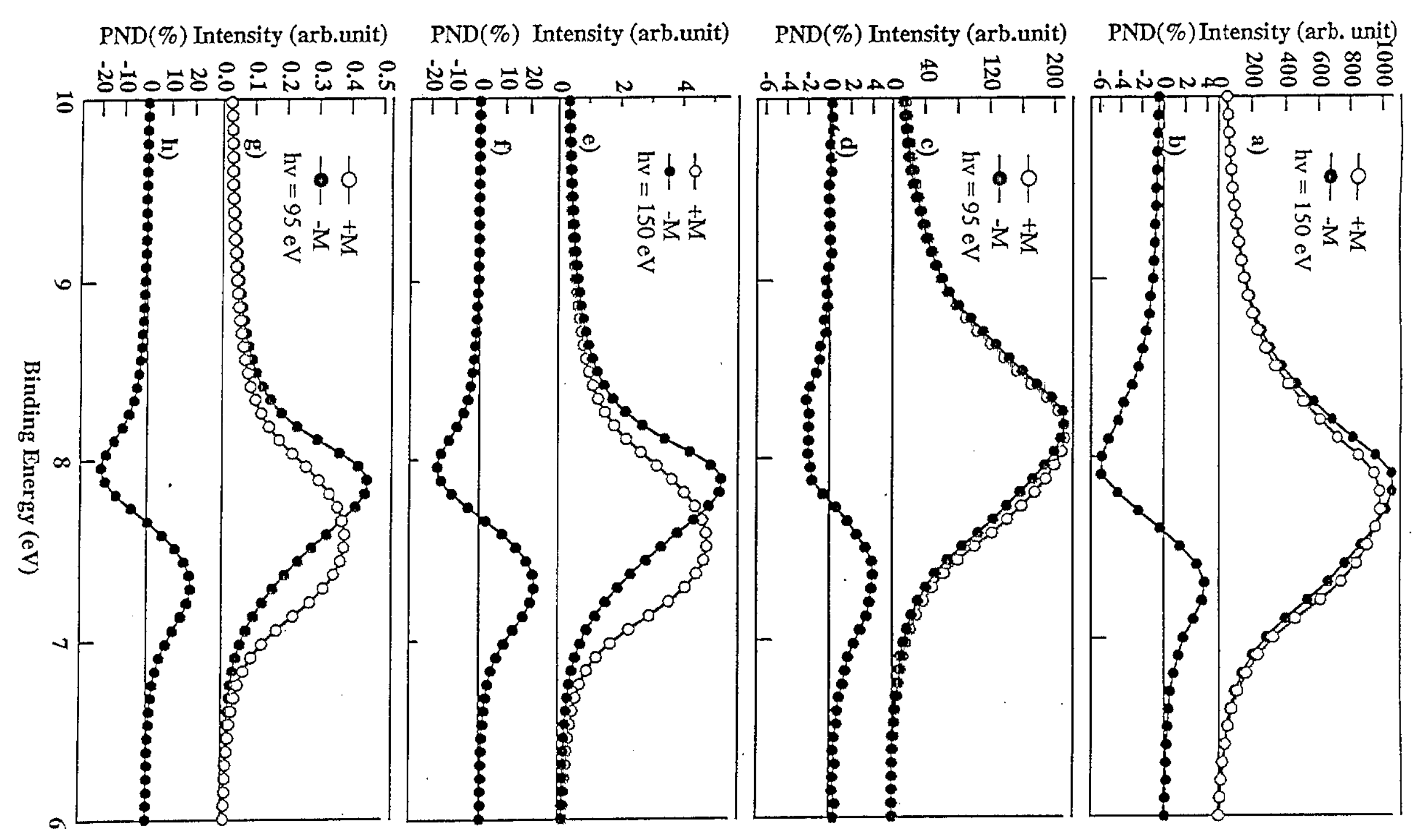



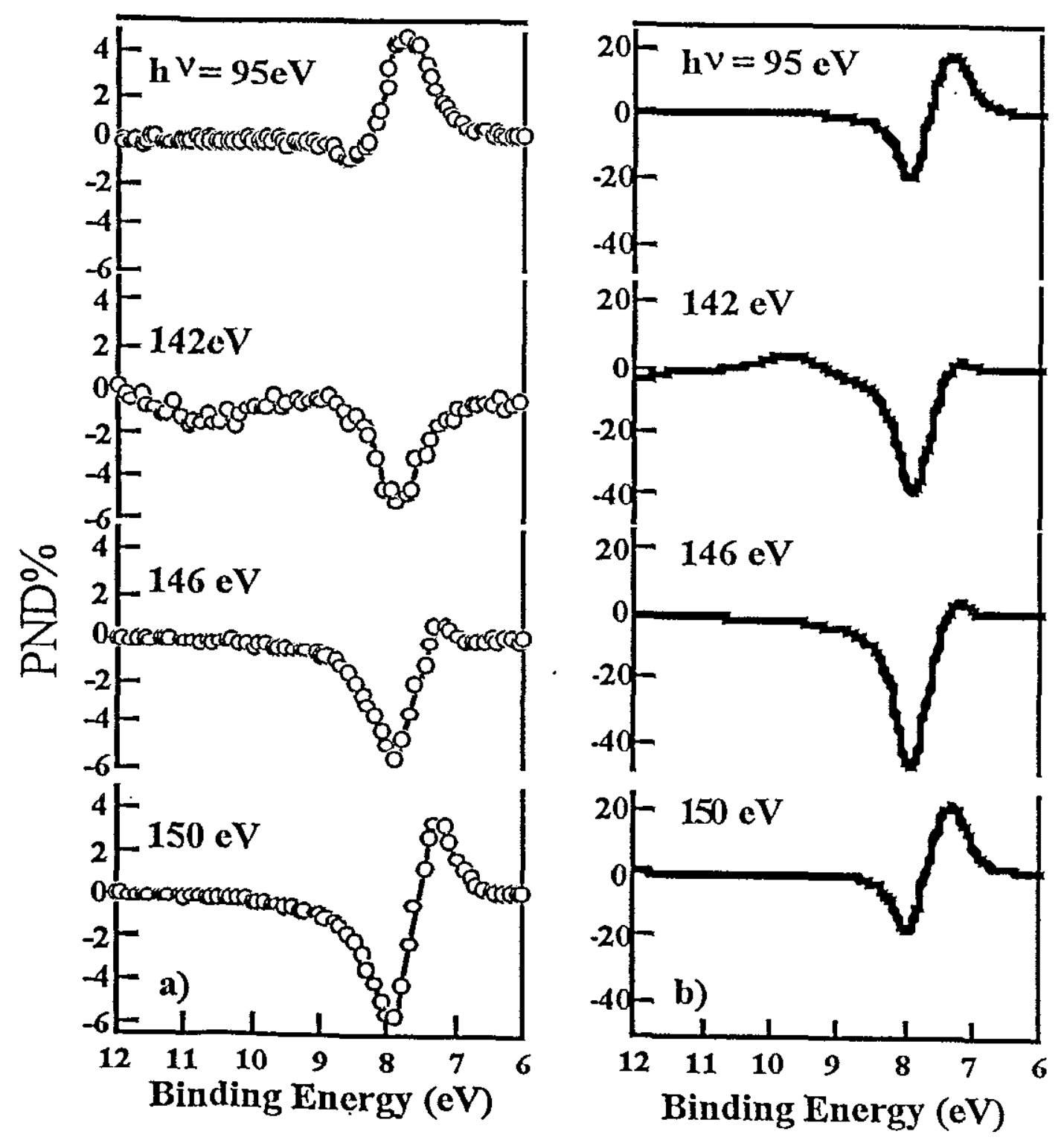

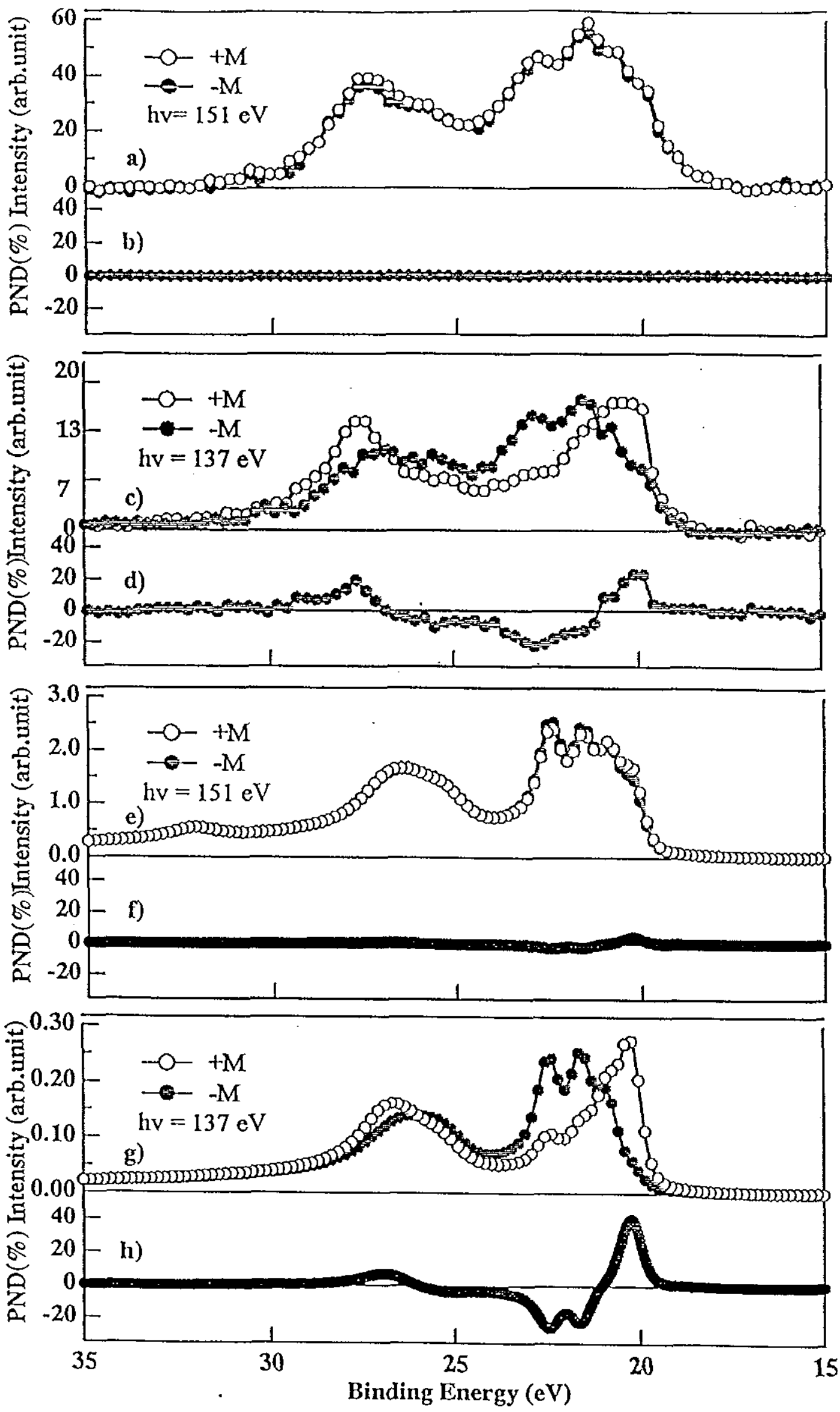